\title{
A personalized robot companion? - The role of individual differences on spatial preferences in HRI scenarios.
}

\author{
Dag Sverre Syrdal, Kheng Lee Koay, Michael L. Walters and Kerstin Dautenhahn \\ Adaptive Systems Research Group, School of Computer Science, University of Hertfordshire, Hatfield, Hertfordshire, UK \\ \{d.s.syrdal, k.1.koay,m.1.walters,k.dautenhahn\}@herts.ac.uk
}

\begin{abstract}
This study investigated the relationship between individual differences and proxemic behaviour in an HRI setting involving a robot approaching a person. In total 33 participants took part in three different scenarios; verbal interaction, physical interaction and no interaction. Participant control over the robot, and approach direction was also varied. Measurements of the preferred robot approach distance was obtained, and analysed along with the participants' demographic and personality data. The results indicate differences in approach direction preferences based on gender. Also, results show that the participants' personality traits of extraversion and conscientiousness are associated with changes in approach distance preferences according to robot autonomy. The results are discussed in light of relevant literature from the social sciences.
\end{abstract}

\section{INTRODUCTION}

The main aim of this study was to investigate individual differences in proxemic behaviour and preferences, i.e. behaviour and preferences related to social distances in an interaction, applied to different Human-Robot Interaction (HRI) scenarios. Existing research in Human-Human interaction indicates that a large part of the variance in such behaviour is caused by factors dependent upon the individual differences in the participants in a given interaction. Therefore, exploring the individual characteristics that may influence such behaviour in HRI scenarios is necessary in order to understand issues related to robot navigation in human-centred environments.

\section{A. Social Robots}

It is expected that robots will be widespread in human centred environments in the near future [1]. This will offer new challenges to researchers in the field of Human-Robot Interaction (HRI). The relationships and dynamics for interaction with such robots will be different, both in the range of interactions, as well as the situations that will occur, to those in public or in the workplace $[2,3]$. For a robot to operate successfully in human-centred environments, it needs to be able to behave in a manner that is socially appropriate. This means that it should perform its functions in a manner that does not upset or disturb any humans it may encounter. In

\footnotetext{
${ }^{1}$ Manuscript received March $9^{\text {th }}, 2007$. The work described in this paper was conducted within the EU Integrated Project COGNIRON ("The Cognitive Robot Companion") and was funded by the European Commission Division FP6-IST Future and Emerging Technologies under Contract FP6-002020
}

this human-centred view, interaction with robots needs to be comfortable and acceptable to potential human users.

An adaptive personalised robot companion [4], must also be capable of adapting to the individual needs and preferences of its users. In order to implement these abilities, the behavioural dimensions of adaptivity as well as the correlates of individual differences in users are necessary topics for investigation. Of particular interest is the use of proxemics research to determine rules of navigation and interaction under different circumstances [5]. Proxemics, a term coined by the anthropologist Edward Hall refers to the study of measurable distances between people as they interact [6]. According to amongst others, Dautenhahn et al. [5], proxemics is a salient issue to study in HRI scenarios. The robots used in these studies do not need to be overly sophisticated: even robots with limited sensory capabilities may be able to use distance data from people in its environment to ensure that its behaviour within a social setting will be deemed appropriate by its users.

Previously, we have explored some of the general issues in proxemics research as it applies to HRI, both as regards interaction distances between robots and humans [7], as well as approach directions for robots moving towards humans [8-11]. Other researchers have also studied issues of proxemics in HRI scenarios, including Yan and Matarić [12] whose findings, from observations of multiple human or robot actors interacting, corresponded with some aspects of Hall's theories. Similar findings were reported by Hüttenrauch et al. [13].

\section{B. Proxemics - A brief overview:}

When investigating proxemics in a HRI setting, it is useful to first consider the role of proxemics in human-human interaction. The term "proxemics" was introduced by Edward Hall and a brief description of this theory and research will follow. In his book, 'The Hidden Dimension' [6], Hall primarily viewed proxemics as the study of differences between cultures. Hall also provided distance data and divided distances in human-human interaction in four categories and attempted to measure these distances for interactions taking place between North Americans. These measurements were obtained primarily from observing naturally occurring dyadic interactions in field studies and lead to the distinctions between Public Distance (3.5 meters and above, Social Distance (1.5-.3.5 meters), Personal Distanc (0.5-1.5 meters ) and Intimate Distance (.05 meters and below). 


\section{Proxemics and HRI}

Hall's distances are meant to apply only to interactions taking part between North Americans, and may have limited relevance to researchers working within other cultures. Aside from this there are two primary issues that limit the usefulness of Hall's categories to HRI research:

The first issue is the lack of a theoretical framework relevant to HRI. Hall's research was primarily concerned with cross-cultural differences. As such it does not offer any insight into the dynamics that control the appropriate proxemic behaviour in any particular HRI situation [14].

The second issue is that the distances described by Hall are not robust, both in terms of accuracy and also generalisability across different individuals and situations. Several researchers have demonstrated that Hall's initial measurements are difficult to replicate, both in experimental settings as well as in field observation [15]. Also, participants have been shown to vary their interaction distances in response to proxemic behaviour of others, both from different (sub) cultures or from within the same culture $[16,17]$. In addition, individual differences such as social status [18], gen$\operatorname{der}[19]$ and personality $[20,21]$ have been shown to impact strongly on proxemic preferences in human-human interactions.

A thorough discussion of the theories and models of proxemics preferences and behaviour $[14,22]$ is beyond the scope of this paper, and so we will concern ourselves with factors that have been shown to impact human-human proxemics in empirical studies. Broadly, we will categorise these into factors that are external to the individual and factors that arise from the individual. As this paper's primary focus is on the contribution of individual differences, these will be discussed in greater detail than external factors. The factors which may have an impact on the proxemic behaviour in any given interaction include:

- External
a. Cultural and sub-cultural norms
b. Situational/interactional context
c. Degree of acquaintance between actors
d. Relative social status between actors

- Internal
e. Gender
f. Personality
g. Physical attributes (e.g. height)
h. Health and medical factors (disabilities etc. that may impact human-human interaction and communication)
i. Other individual differences

In the following we limit the discussion of internal factors to $\mathrm{e}$ and $\mathrm{f}$ which have been addressed in our study.

\section{Extemal Factors.}

a) Cultural and Sub-cultural norms:

Cross-cultural generalisability of results from HRI trials may be made difficult as not only may norms for proxemic differ, but attitudes towards robots have been shown to vary from culture to culture [23].

\section{b) Situational/Interactional Context:}

Widely reported in studies on human proxemic behaviour [14], it is only natural to assume that this factor may have a strong impact on proxemic preferences when interacting with a robot. Our initial studies on human-robot proxemics indicated that many participants were comfortable approaching a stationary robot (a large minority even to within Hall's "Intimate space" zone found for human-human social distances) but the results were less clear for the condition where the robot actually approached them under the robot's control [24].

\section{c) Degree of Acquaintance:}

According to Hall [6], this is one of the most important factors in intra-cultural proxemic variation. Our previous HRI studies have used naïve participants, interacting with a robot for the first time, and so the results from these studies may not be applicable to the preferences of users who have interacted with a robot for a prolonged period of time.

\section{d) Social Status:}

Assigning a human concept as social status to a robot may be problematic. However, it may be possible to investigate whether or not anthropomorphic attributions [25] to a robot will influence proxemic preferences on the part of the user. Another means to manipulate this is to change the degree of displayed autonomy by the robot. Kim \& Hinds [26] argue that the perception of a robot's role in an interaction changes depending on its degree of autonomy, and as such its status in a social interaction may change.

\section{Internal Factors}

\section{e) Gender}

The topic of gender in proxemic research has been studied quite extensively $[19,27]$, by researchers from various fields including ethology and socio-biology. These theories argue that males are more territorial than females and so will be more likely to have a larger personal space. Males seem to tend to respond more strongly to intrusions into this space. As such intrusions may trigger fight or flight responses to a larger degree than for females. However, Gillespie and Leffler [14] argue in their review of proxemics research that it is very difficult to establish a clear difference between behaviour arising from genetically determined biological processes and behaviour arising from socialization within a given culture. This is also an issue in general for the socio-biological and ethological understanding of behaviour [28], but any attempt to disentangle this relationship is beyond the scope of our investigation. Despite this issue, systematic differences have been observed between males and females in proxemic preferences and behaviour. As such it may well have an impact on HRI scenarios.

\section{f) Personality and Individual Differences}

This paper is primarily concerned with the role of individual differences on proxemic behaviour and preferences in HRI 
studies. Individual differences have been considered to be a factor in regulating proxemic preferences and behaviour in human-human interactions $[21,29,30]$. We have also considered these issues in two previous HRI studies. Walters et al. [7], studied the impact of personality on preferred distances to a robot and found that "proactive" 1 participants preferred to keep a larger distance between themselves and the robot. Syrdal et al. [8] found a small proxemic tolerance effect for extraversion, namely, extraverts rated approach directions that were rated as least comfortable by the overall sample, as more comfortable than other participants. A similar effect was found by Gockley and Mataric [31], who reported an overall greater tolerance for robot proximity among extroverts in a stroke rehabilitation task.

The model of personality used in the current study is the Big Five Model, measured using the Big Five Domain Scale from IPIP [32]. Please see table 1 for a brief overview of the personality factors described in this particular model.

TABLE 1 BIG FIVE PERSONALITY FACTORS ( ADAPTED FROM[33])

\begin{tabular}{ll}
\hline \hline Emotional Stability & $\begin{array}{l}\text { Anxiety, depression, self-consciousness, im- } \\
\text { pulsiveness, vulnerability }\end{array}$ \\
& Warmth, gregariousness, assertiveness, activity, \\
excitement seeking, positive emotions & Trust, straightforwardness, altruism, compli- \\
ance, modesty, tender-mindedness & Competence, order, dutifulness, achievement \\
Conscientiousness & striving, self-discipline, deliberation \\
Intellect & Imagination, ideas, abstract thought, range of \\
& interests \\
\hline
\end{tabular}

\section{METHOD}

\section{A. Participants:}

The 33 participants (20 males and 13 females, sample ${ }_{W}$ ) were drawn from staff and students at the University of Hertfordshire, recruited through an advertisement on the university's intranet. The age range in the sample varied from 18 to 50 , with a median age of 23 . Consent for participation was obtained in writing. Twelve participants were selected for the longitudinal study (sample $e_{L}$ ). From these, 4 were female and 8 were male, the age range was 21 to 40 with a median age of 23 . They were paid a modest compensation in order to reinforce their continued participation throughout the 6 week period.

Apparatus:

The robots used for this particular study were two Peoplebots $^{\text {tm }}$ (commercially available research robot platforms from ActivMedia Robotics). The robots were operated using the Wizard of Oz Method [9]. In addition, two video cameras recorded the experimental HRI sessions. To record participants' approach distance preferences, a Comfort Level Device (CLD) was used. This device is described in detail in Koay et al. [34]. The experiment took place in the living room of the University of Hertfordshire "Robot House", a domestic home-environment, in order to provide a more ecologically valid context.

\footnotetext{
${ }^{1}$ Please see Walters et al. [7] for a full description of the personality traits used in this study. In brief, proactiveness is a trait that shares correlates to some extent with both extraversion and psychoticism.
}

Experimental Procedure:

For each experimental condition, the robot approached the participants from across the room. The participants were instructed to press a button on the CLD when they felt they did not want the robot to approach any further during each trial run. There were three repeated measures variables for this experiment, making it a $2 \times 3 \times 2$ repeated measures design. The conditions were as follows (cf. [35] for a more exhaustive description of the scenario types and experimental conditions):

- Human in Control/Robot in Control Variable

a. Human in Control ( $\mathrm{HiC}$ - Pressing the CLD button would stop the robot from approaching further

b. Robot in Control ( $\mathrm{RiC}$ ) - Pressing the CLD had no impact on the robot's approach and was only used to record preferences.

- Interaction Variable

a. No Interaction - Robot would just approach the participant before turning around and moving away

b. Verbal Interaction - Robot approached participant in order for him or her to practice verbal movement commands

c. Physical Interaction - Robot approached the participant with three upturned cups in its tray. Participant would then need to look under each cup in turn in order to find a wooden cube

- Direction variable

a. Robot would approach directly from the front.

b. Robot would approach from the front right of the participant (see fig 3 in [8] for diagram of the directions).

In addition, the experiment also used robots with four different appearances. These were categorized as Short Mechanical, Short Humanoid, Tall Mechanical and Tall Humanoid. Each participant was exposed to all the different repeated measures conditions but only interacted with one of the four robot appearances. In addition, each participant completed a brief demographics questionnaire as well as the Big Five Domain Scale. The participants were also given a questionnaire for them to indicate what interaction types they most preferred. These results were analysed along with the CLD data.

The long-term participants were exposed to the experiment on approach distances 3 times: Week 1 (first encounter with the robots, sample $W$ ), Week 2 (in order to control for the novelty effect of an initial interaction, sample s. $_{\text {), and finally }}$ Week 5 (after having been exposed to the robot in various

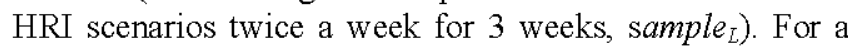
more detailed explanation of the experimental setup, and the intermediate scenarios, please refer to [35].

\section{RESULTS}

\section{A. General Results}

1) CLD Data:

A detailed overview of the distance data can be found in [35], however, a general overview will be given here.

There was a significant main effect for Robot Appearance, and pair wise comparisons found that participants allowed the robots with mechanical appearance to approach to a closer distance than those with a humanoid appearance. For 
the short-term experiment, there was a significant main effect for interaction type. Subsequent pair-wise comparisons found that participants preferred the robot to approach closer for physical interaction than for verbal or no interaction. There was also a significant main effect for approach direction. The pair-wise comparison showed that participants in general allowed the robot to approach closer if it was approaching from the side rather than directly from the front.

Examining the long-term effects, we found no significant differences between week 1 and 2, however, participants allowed the robot to approach significantly closer in week 5 , pointing to an habituation effect in spatial preferences due to the prolonged interaction period, independent of any novelty effect (for details of this habituation effect see [36]). There were no systematic effects for the Human in Control and Robot in Control (HiC/RiC) conditions.

\section{1) Questionnaire Data:}

While the CLD data did not show a significant difference dependent on $\mathrm{HIC} / \mathrm{RIC}$ condition, the post-session questionnaire indicated that there was some impact on participant preferences as to preferred interaction type. Please see Table 2 for an overview. It seems that the long-term participants in weeks 2 and 5 preferred the verbal interaction scenario to the physical interaction scenario in the $\mathrm{RiC}$ condition, while this was not the case in the $\mathrm{HiC}$ condition.

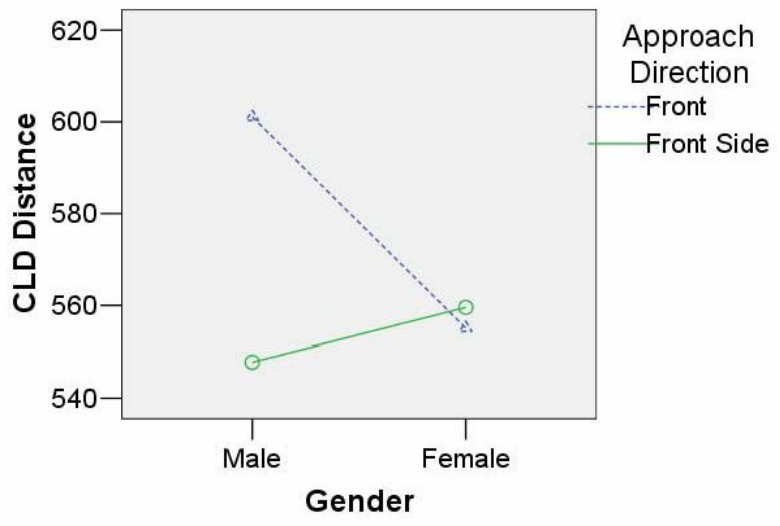

Figure 1. Interaction effect for gender and approach direction.

\section{B. Demographics:}

No significant effects in approach distance preferences were found for age, academic background or computer proficiency. However, a mixed ANOVA found a significant interaction effect in Week 1 between gender and approach direction $(\mathrm{F}(1,31)=4.515, \mathrm{p}<.05)$. The effect is described below in figure 1 .

Figure 1 shows that for females, there is no difference in preferred approach distances for the two different approach directions. For males, however, there is a clear difference between the two directions, where males allow the robot to
TABLE 2

QuestionNaIRE RESUlts For THE MOST

COMFORTABLE INTERACTION SCENARIO

\begin{tabular}{l|l|l|l|l|l|l|l|l}
\hline \hline & \multicolumn{2}{|l|}{$\begin{array}{l}\text { Wk. 1 } \\
\left(\text { Sample }_{W}\right)\end{array}$} & \multicolumn{2}{|l|}{$\begin{array}{l}\text { Wk. 1 } \\
\left(\text { Sample }_{L}\right)\end{array}$} & \multicolumn{2}{|l|}{$\begin{array}{l}\text { Wk. 2 } \\
\left(\text { Sample }_{L}\right)\end{array}$} & \multicolumn{2}{|l}{$\begin{array}{l}\text { Wk. 5 } \\
\left(\text { Sample }_{L}\right)\end{array}$} \\
\cline { 2 - 10 } & HiC & RiC & HiC & RiC & HiC & RiC & HiC & RiC \\
\hline $\begin{array}{l}\text { No } \\
\text { Interaction }\end{array}$ & 2 & 4 & 2 & 1 & 3 & 2 & 1 & 2 \\
\hline $\begin{array}{l}\text { Verbal } \\
\text { Interaction }\end{array}$ & 16 & 14 & 5 & 6 & 5 & 7 & 4 & 7 \\
\hline $\begin{array}{l}\text { Physical } \\
\text { Interaction }\end{array}$ & 15 & 13 & 5 & 4 & 3 & 2 & 5 & 1 \\
\hline $\begin{array}{l}\text { No Re- } \\
\text { sponse }\end{array}$ & 0 & 2 & 0 & 1 & 1 & 1 & 2 & 2 \\
\hline Total & 33 & 33 & 12 & 12 & 12 & 12 & 12 & 12 \\
\hline \hline
\end{tabular}

approach closer when the robot is approaching from the side than when it is approaching from directly from the front.

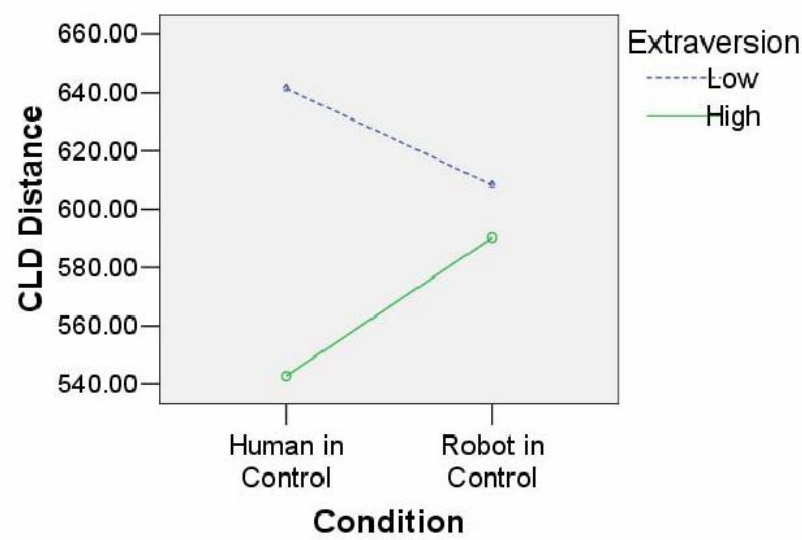

Figure 2. Extraversion, $\mathrm{HiC} / \mathrm{RiC}$ Condition and Approach distance preferences

\section{B. Personality:}

A mixed model ANOVA found no significant main effects for personality and overall preferences for approach distances across the conditions. However, a significant interaction effect for extraversion and the $\mathrm{HiC} / \mathrm{RiC}$ condition $(\mathrm{F}(1,14)=7.43, \mathrm{p}<.05)$ as well as for conscientiousness and the $\mathrm{HiC} / \mathrm{RiC}$ condition $(\mathrm{F}(1,14)=5.73, \mathrm{p}<.05)$ was found. See Figures 2 and 3 for a description of these effects.

Figure 2 suggests that there is a clear difference between participants with low and high extraversion scores in the Human in Control condition. Participants scoring high on extraversion allowed the robot to come closer in the Human in Control condition than participants with a low extraversion score. However, for the Robot in Control condition the difference between the two groups is much smaller. This suggests that introverts were comfortable with the robot coming closer in the $\mathrm{RiC}$ condition, while Extraverts were less comfortable with the robot approaching closely in this condition.

The opposite seems to be the case for conscientiousness. Participants scoring low on conscientiousness allowed the robot to approach more closely in the $\mathrm{HiC}$ condition than they were comfortable with for the $\mathrm{RiC}$ condition. There was no marked difference due to $\mathrm{HiC} / \mathrm{RiC}$ conditions for participants scoring high on conscientiousness. 
As mentioned above with regard to gender, the low sample size made it difficult to investigate any long-term effects for personality. No discernible trends were found in the long-term sample for personality.

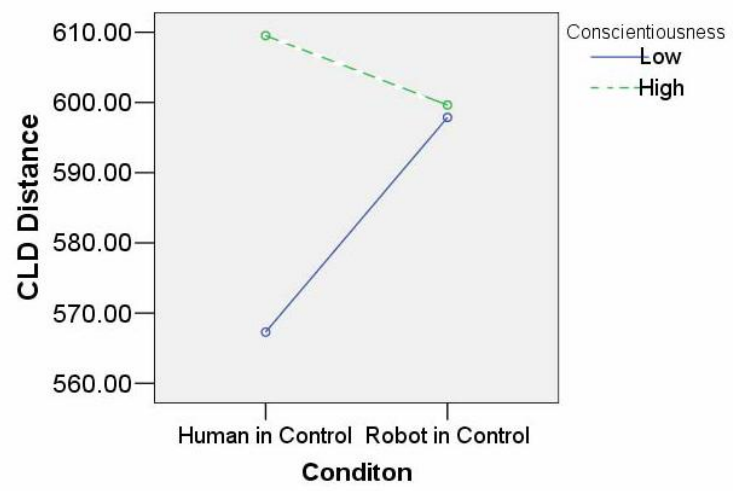

Figure 3. Conscientiousness, HiC/RiC Condition and Approach Distance Preferences

\section{Discussion}

\section{A. The Role of Gender}

The results presented here indicate that, while it does not significantly impact on overall distance preferences, gender does play a role in determining proxemic behaviour and preferences in a HRI scenario. While there were no difference in preferred approach distances between males and females when the robot approached from the side, females allowed the robot to approach closer than males when it was approaching directly from the front. Our previous studies [8, 10] have also shown that while most participants (on initial encounters) found the front direct approach to be less comfortable than from the side approaches (as this approach felt more confrontational and threatening), a minority of females actually preferred a frontal approach. Kendon [36] in his work on geometrical patterns (F-formations), formed in human-human interactions, observes that approaches or interactions when both individuals are facing each other directly tend to be interactions that are confrontational and/or competitive in nature. The proxemic preferences displayed by the male portion of the sample for this approach direction may indicate that males perceived the robot's behaviour as a territorial threat to much greater degree than females and therefore responded by preventing it from approaching further. This could be caused by more territorial behaviour in the male sample, or different coping strategies for invasions of personal space in males and females. It may also reflect a gender difference in how participants perceive the robot as a social actor. The long-term results also suggest that gender differences are not as relevant in long-term interactions as they are in initial interactions.

\section{B. Personality}

While the $\mathrm{HiC} / \mathrm{RiC}$ conditions did not produce a significant main effect in the short-term sample, they did interact significantly with extraversion and conscientiousness, indicat- ing that the two traits are related to participants' reactions to perception of control and autonomy across the scenarios.

Participants scoring high in extraversion allowed the robot to approach closer in the Human in Control condition than they preferred in the Robot in Control condition, while there were no difference between these conditions for participants with low extraversion scores. This would indicate that participants with higher extraversion scores put a greater emphasis on control than the rest of the sample. Literature on personality and proxemics do tend to indicate that extroverts are more tolerant to invasions of personal space than introverts [21] and our previous research has shown this to be the case in HRI scenarios as well [8]. In light of this, the results seem to be counterintuitive. One explanation for this result may be that extraversion shares correlates with the personality construct of dominance [33], a construct that has been shown to be related to initiation of close distance interactions [37], but also is associated with a preference for control of such interactions. This could be a possible cause of this effect.

For conscientiousness, the effect was similar to that of Extraversion. Participants scoring low in conscientiousness allowed to robot to approach closer in the $\mathrm{HiC}$ condition than they preferred for the RiC condition, while no difference between conditions was found for participants with higher conscientiousness scores. There are several explanations that could account for this. The first is that individuals with high conscientiousness scores tend to be more consistent in their behaviour and are less likely to change their preferences than those with low scorers, thus accounting for the lack of change between these conditions. Also, individuals with low conscientiousness often exhibit less impulse control, which also may have led them to press the CLD earlier in a situation in which they were not in control of the robot's behaviour.

While future research with larger sample sizes and a variety of HRI scenarios will need to verify the above interpretation of the results, the time and resources necessary to conduct such large-scale studies mean that research questions and measurements need to be informed by smaller studies like this one in order to make best use of these resources.

\section{Conclusions and Future Research}

This study has shown that individual differences due to gender and personality have an impact on proxemics preferences in HRI situations, just as they do in human-human interactions. What is less clear from these results is how these individual differences affect proxemics preferences in the long-term. What does seem to be the case, however, is that the impact of individual differences change as participants become more familiar with robot.

Future research should endeavour to integrate results from behavioural responses in HRI scenarios with participant evaluation of the scenario in order to gain a fuller picture of the mechanisms that govern the relationships between individual differences and proxemic preferences and behaviour. This will enable further investigation of the dimensions of 
adaptivity that will be necessary for a robot to navigate human-centred environments in a socially acceptable manner.

\section{REFERENCES}

[1] W. Gates, "A robot in every home," Scientific American, 2007

[2] J. Goetz and S. Kiesler, "Cooperation with a Robotic Assistant," CHI '02 Conference on Human Factors in Computing Systems, 2002.

[3] P. Wu and C. Miller, "Results from a field study: The need for an emotional relationship between the elderly and their assistive technologies," in Foundations of Augmented Cogniton, Vol 11. Mahwah: Lawrence Erlbaum Assoc Publ, 2005, pp. 889-898.

[4] K. Dautenhahn, "Robots We Like to Live With? - A Developmental Perspective on a Personalized, Life-Long Robot Companion," Proceedings of the 13th IEEE International Workshop on Robot and Human Interactive Communication(RO-MAN 2004), pp. 17-22., 2004.

[5] K. Dautenhahn, B. Ogden, and T. Quick, "From Embodied to Socially Embedded Agents - Implications for Interaction-Aware Robots," Cognitive Systems Research, vol. 3, pp. 397-428, 2002.

[6] E. T. Hall, The Hidden Dimension. New York: Doubleday, 1966. [7] M. L. Walters, K. Dautenhahn, R. T. Boekhorst, K. L. Koay, C. Kaouri, S. Woods, C. Nehaniv, D. Lee, and I. Werry, "The influence of subjects' personality traits on personal spatial zones in a human-robot interaction experiment," Proc. 14th IEEE International Workshop On Robot And Human Interactive Communication (RO-MAN 2005), pp. 347-352, 2005.

[8] D. S. Syrdal, K. Dautenhahn, S. Woods, M. L. Walters, and K. L. Koay, "'Doing the right thing wrong' - Personality and tolerance to uncomfortable robot approaches," Proc. 15th IEEE International Workshop on Robot and Human Interactive Communication(RO-MAN 2006), pp. 183-188, 2006.

[9] M. Walters, S. Woods, K. L. Koay, and K. Dautenhahn, "Practical and methodological challenges in designing and conducting interaction studies with human subjects.," AISB'05 Symposium on Robot Companions Hard Problems and Open Challenges in Human-Robot Interaction, pp. 110-120, 2005.

[10] S. Woods, M. Walters, K. L. Koay, and K. Dautenhahn, "Comparing Human Robot Interaction Scenarios Using Live and Video Based Methods: Towards a Novel Methodological Approach," Proc. AMC'06, The 9th International Workshop on Advanced Motion Control, Istanbul March 27-29, pp. 750-755, 2006.

[11] S. N. Woods, M. L. Walters, K. L. Koay, and K. Dautenhahn, "Methodological Issues in HRI: A Comparison of Live and Video-Based Methods in Robot to Human Approach Direction Trials," Proceedings, 15th IEEE International Workshop on Robot and Human Interactive Communication (RO-MAN2006), pp. 51-58, 2006.

[12] H. Yan and M. J. Mataric', "General spatial features for analysis of multi-robot and human activities from raw position data,"

Proceedings of the 2002 IEEE/RSJ International Conference on Intelligent Robots and Systems (IROS'02), pp. 2770-2775, 2002.

[13] H. Huettenrauch, K. S. Eklundh, A. Green, and E. A. Topp, "Investigating Spatial Relationships in Human-Robot Interaction," Proceedings of the IEEE/RSJ International Conference on Intelligent Robots and Systems(IROS 2006), Beijing, China, pp. 5052-5059, 2006.

[14] D. L. Gillespie and A. Leffler, "Theories of nonverbal behavior: A critical review of proxemics research," in Sociological Theory Vol 1., 1983, pp. 120-154.

[15] H.W. Smith, "Territorial Spacing on a beach revisited: A Cross-National Exploration," Social Psychology Quarterly, vol. 44, pp. 132-137, 1981.

[16] C. Albas, "Proxemic Behavior: A Study of Extrusion," Joumal of Social Psychology, vol. 131, pp. 697-702, 1991.

[17] C. Albas and D. Albas, "Aligning Actions: The case of subcultural proxemics," Canadian Ethnic Studies, vol. 21, pp. 74-82, 1989.

[18] A. Leffler, D. L. Gillespie, and J. C. Conaty, "The Effects of Status Differentiation on Nonverbal Behavior," Social Psychology Quarterly, vol. 45, pp. 153-161, 1982.
[19] K. A. Krail and G. Leventhal, "The Sex Variable in the Intrusion of Personal Space," Sociometry, vol. 39, pp. 170-173, 1976.

[20] L. Eastwood, "Personality, Intelligence and Personal Space among Violent and Nonviolent Deliquents," Personality and Individual Differences, vol. 6, pp. 717-723, 1985.

[21] J. L. Williams, "Personal space and its relation to extraversion-introversion," Canadian-Journal-of-Behavioural-Science, vol. 3, pp. 156-160, 1971.

[22] P. A. Andersen, L. K. Guerrero, D. B. Buller, and P. F. Jorgensen, "An Empirical Comparison of Three Theories of Nonverbal Immediacy Exchange," Human Communication Research, vol. 24, pp. 501-535, 1998.

[23] C. Bartneck, T. Nomura, T. Kanda, T. Suzuki, and K. Kennsuke, "A cross-cultural study on attitudes towards robots.," Proceedings of the HCI International 2005, Las Vegas., 2005.

[24] M. L. Walters, K. Dautenhahn, K. L. Koay, C. Kaouri, René te Boekhorst, C. Nehaniv, I. Werry, and D. Lee, "Close Encounters: Spatial Distances between People and a Robot of Mechanistic Appearance," Proceedings of 2005 5th IEEE-RAS International Conference on Humanoid Robots, Tsukuba, Japan, pp. $450-455,2005$

[25] D. S. Syrdal, K. Dautenhahn, S. Woods, M. Walters, and K. L. Koay, "Looking Good? Appearance Preferences and Robot Personality Inferences at Zero Acquaintance," Proceedings of 2007 AAAI Spring Symposia, In Press.

[26] T. Kim and P. Hinds, "Who should I blame? Effects of Autonomy and Transparency on Attribution in Human-Robot Interaction," Proc. 15th IEEE International Workshop on Robot and Human Interactive Interactive Communication(RO-MAN 2006), pp. 80-85, 2006.

[27] K. G. Bailey, J. J. Hartnett, and F. W. Gibson, "Implied threat and the territorial factor in personal space," Psychological Reports, vol. 39, pp. 263-270, 1972.

[28] A. Giddens, Sociology, 5 ed. Cambridge: Polity, 2006.

[29] Y. Bar Haim, O. Aviezer, Y. Berson, and A. Sagi, "Attachment in infancy and personal space regulation in early adolescence," Attachment-and-Human-Development, vol. 4, pp. 68-83, 2002.

[30] M. J. Strube and C. Werner, "Personal space claims as a function of interpersonal threat: The mediating role of need for control," Joumal of Nonverbal Behavior, vol. 8, pp. 195, 1984.

[31] R. Gockley and M. J. Mataric', "Encouraging Physical Therapy Compliance with a Hands off Mobile Robot," Proc. HRIO6, Salt Lake City, Utah, USA, 2006, pp. 150-155, 2006.

[32] L. R. Goldberg, " A broad-bandwidth, public domain, personality inventory measuring the lower-level facets of several five-factor models.," Personality Psychology in Europe, vol. 7 , pp. 7-28, 1999.

[33] G. Matthews, I. J. Deary, and M. C. Whiteman, Personality Traits. Cambridge, UK: Cambridge University Press, 2003.

[34] K. L. Koay, M. L. Walters, S. N. Woods, and K. Dautenhahn, "Empirical Results from Using a Comfort Level Device in Human-Robot Interaction Studies.," Proceeds of HRI06, pp. 194-201, 2006.

[35] K.-L. Koay, D. S. Syrdal, M. L. Walters, and K. Dautenhahn, "Longditudinal Studies in HRI," Submitted manuscript, Submitted.

[36] A. Kendon, Conducting interaction - Patterns of behavior in focused encounters. Studies in interactional sociolinguistics. Cambridge, NY, USA: Press syndicate of the University of Cambridge, 1990.

[37] J. K. Burgoon and S. B. Jones, "Toward a Theory of Personal Space Expectations and Their Violations," Human Communication Research, vol. 2, pp. 131-146, 1976. 